\title{
D-ADMM Based Distributed MPC with input-output models*
}

\author{
Rafael P. Costa ${ }^{1}$, João M. Lemos ${ }^{2}$, João F. C. Mota ${ }^{3}$ and João M. F. Xavier ${ }^{4}$
}

\begin{abstract}
This article presents a distributed model predictive controller (MPC) based on linear models that use input/output plant data and D-ADMM optimization. The use of input/output models has the advantage of not requiring a Kalman filter to estimate the plant state. The D-ADMM algorithm solves the optimization problem associated to a cost function that is the sum of the control agents private costs, being a modification of the Alternating Direction of Multipliers (ADMM) algorithm that requires no central node and implies a significant reduction in the communication among adjacent nodes. The distributed MPC is obtained for the special case of a linear graph. An application to distributed control of a water delivery canal is presented to illustrate the algorithm.
\end{abstract}

\section{INTRODUCTION}

\section{A. Motivation and review}

The development of distributed versions of model predictive control (D-MPC) is currently the subject of considerable interest. A recent collection of existing algorithms [1] listed 35 different approaches. In addition, recent works on the topic include as significant examples [2], [3], [4], [10], [13], [5], [7], [8]. While many applications can be considered, such as the control of power networks [6] or electrical vehicle charging control [9], this article concentrates its attention on the control of water delivery canals. Besides its socioeconomic impact, this problem raises interesting control problems that have been addressed by distributed predictive control [14], [15], [11], as well as with other approaches.

Water delivery canals are made of a sequence of reaches, delimited by gates that control the water flow. Some of these structures extend over wide areas, sometimes over different countries or different administrative regions. This large spatial scale motivates the use of distributed control, where local controllers, associated to gates, exchange information about their decisions via a communication network. In the situation considered in this article, it is assumed that each local control agent is only able to negotiate the value of its manipulated variable with its direct neighbors.

A recent approach to obtain distributed controllers that is suitable for water delivery canals is based on Game theory

\footnotetext{
*This work was supported by FCT under contracts PTDC/EMSCRO/2042/2012 (project ORCHESTRA Distributed Optimization and Control of Large Scale Water Delivery Systems) and PEstOE/EEI/LA0021/2013.

${ }^{1}$ Rafael Costa is with INESC-ID, Lisboa, Portugal albert.authorepapercept. net

${ }^{2}$ João M. Lemos is with INESC-ID/Instituto Superior Técnico, Universidade de Lisboa, R. Alves Redol 9, 1000-029 Lisboa, Portugal jlmleinesc-id.pt

${ }^{2}$ João Mota was with ISR/Instituto Superior Técnico, Universidade de Lisboa, Lisboa, Portugal j.mota@isr.ist.utl.pt

${ }^{2}$ João Xavier is with ISR/Instituto Superior Técnico, Universidade de Lisboa, Lisboa, Portugal jxaviereisr.ist.utl.pt
}

[10], [8], [11], [12]. Although it leads to algorithms that are easy to implement, leading to good results, if proper actions are not taken, these methods have the drawback of converging to a Nash equilibrium, or to a modification of a Nash equilibrium, that may be far away from the global minimum that would be obtained if a centralized controller is used.

In order to avoid the above problem, one may resort to methods based on convex optimization and duality [16]. Accordingly, in the so-called decomposition phase, the whole system is first decomposed in subsystems, each one provided with a replica of all the manipulated variables. Then, in the coordination phase, a sequence of optimization problems is solved in which, in addition to the operational constraints, there is a coordination constraint that imposes that the replicas of the manipulated variables obtained by local control agents are consistent among them. However, solving these problems using straight lagrangian optimization leads to algorithms that require strong assumptions to converge that limit the class of problems to which they can be useful. The Alternating Direction Method of Multipliers (ADMM) is a family of algorithms that uses a lagrangian function augmented with a quadratic term, and perform optimization in alternation along each of the Lagrange multipliers. Although the origin of the method is old [17], it is receiving increasing attention for a variety of applications [19]. However, some forms of the ADMM algorithm, such as described in [19], actually assume a central node, a fact that prevents its use in pure distributed optimization algorithms. In [20], [21] forms of ADMM that do not require this central node (the first with the dual variables associated to nodes, and the second to edges) are presented. These algorithms are named D-ADMM to stress the fact that they do not require a central node, being therefore adequate to solve distributed optimization problems. In [21], the D-ADMM algorithm is optimized such as to reduce the communication required among network nodes.

The use of decomposition-coordination algorithms, together with an augmented lagrangian, has been already used in relation to optimal/predictive control of water delivery canal systems [18], [15]. In this paper, instead, the D-ADMM algorithm presented in [21] is used to obtain a distributed MPC for distributed plants with a linear graph.

\section{B. Contributions and organization}

The contribution of this paper consists of a distributed model predictive controller (MPC) based on linear models based on input/output plant data and D-ADMM optimization, and its application to the distributed control of a water 


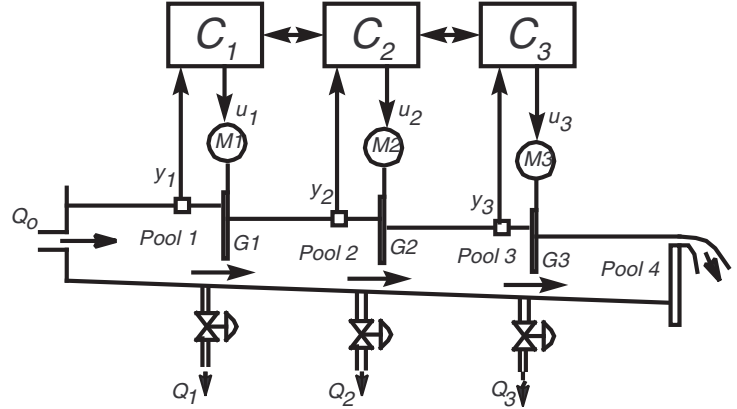

Fig. 1. A schematic view of the canal with the distributed controller.

delivery canal. The paper is organized as follows: After this introductory section, in which the problem is motivated, the most relevant literature is reviewed and paper contributions and organization are stated, the problem is formulated in section II. Predictive models based on input/output data for the class of distributed plant considered are described in section III. Centralized MPC based on input/output models are obtained in section IV and section $\mathrm{V}$ describes the proposed distributed MPC algorithm. The application to a water delivery canal is presented throughout sections II until V. Finally, section VI draws conclusions.

\section{PROBLEM FORMULATION}

Consider a distributed plant made of local plants such that each one interacts only with its neighbors. This plant can be represented by a linear graph in which the nodes are numbered sequentially from 1 to $N_{P}$ and represent the different local plants with local models denoted $\Sigma_{1}, \ldots, \Sigma_{P}$, and the edges are associated to the interactions among neighbor local plants. Each plant $\Sigma_{i}$ has a local manipulated variable denoted $u_{i}$ and a local output $y_{i}$. Both $u_{i}$ and $y_{i}$, for $i=1, \ldots, N_{P}$ are not available centrally, but it is assumed that there is a communication system with a structure that parallels the plant graph, and that allows the local control agents of neighbor plants to exchange information. Furthermore, it is assumed that the local plants interact only through their inputs, meaning that the output of $\Sigma_{i}$ is influenced by $u_{i-1}, u_{i}$ and $u_{i+1}$ (whenever these variables exist).

Associated to each local plant $\Sigma_{i}$ there is a local quadratic cost $J_{i}$ defined by

$$
J_{i}=\sum_{j=1}^{T}\left[\left(y(k+j)-r_{i}(k+j)\right]^{2}+\rho_{i} u_{i}^{2}(k+j-1),\right.
$$

where $k \in \mathbb{N}$ is the current time, $r_{i}$ is the reference to be tracked by $y_{i}$, and $\rho_{i}$ is a weight in the manipulated variable. The overall cost is given by

$$
J=\sum_{i=1}^{N_{P}} J_{i} .
$$

To each local plant $\Sigma_{i}$ a local control agent $K_{i}$ is associated. The objective consists in the cooperation of the local control agents, with the communication limitation expressed above, in order to find the values of the local manipulated variables such that the minimum of the global cost $J$ is tightly approximated, while satisfying at all times $k$ the constraints given by

$$
\begin{aligned}
& u_{i}^{\text {min }} \leq u_{i}(k) \leq u_{i}^{\max }, \\
& y_{i}^{\text {min }} \leq y_{i}(k) \leq y_{i}^{\text {max }},
\end{aligned}
$$

and

$$
S_{R}^{\min } \leq u_{i}(k)-u_{i}(k-1) \leq S_{R}^{\max },
$$

where $u_{i}^{\min }, u_{i}^{\max }, y_{i}^{\min }, y_{i}^{\max }, S_{R}^{\min }$ and $S_{R}^{\max }$ are parameters that define the acceptable operating regime envelope.

Furthermore, the cost minimization is to be made in a receding horizon sense. Each local control agent has a replica of the manipulated variables of its neighbors. At the beginning of each sampling interval, neighbor local control agents exchange information such as to reach a consensus on the values of the manipulated variables.

\section{PREDICTIVE MODELS}

To define the notation, we start by reviewing standard results for predictive models for an isolated local plant. For simplicity, the index of the local system is omitted. The result is then generalized to obtain predictors to the overall plant.

\section{A. Isolated systems}

Consider an isolated system described by the deterministic autoregressive model

$$
A^{*}\left(q^{-1}\right) y(k+1)=B^{*}\left(q^{-1}\right) u(k),
$$

where $q^{-1}$ is the backward shift operator,

$$
A^{*}\left(q^{-j}\right)=1+a_{1} z^{-1}+\cdots+a_{n} z^{-n}
$$

and

$$
B^{*}\left(q^{-j}\right)=b_{0}+b_{1} z^{-1}+\cdots+b_{m} z^{-m} .
$$

Define the polynomials $F_{j}^{*}$, of order $j-1$, and $G_{j}^{*}$, of order $n-1$ to be a solution of the diophantine equation

$$
A^{*}\left(q^{-j}\right) F_{j}^{*}=1-q^{-j} G_{j}^{*} .
$$

Multiplying (6) by $F_{j}^{*}$ and using (9), yields

$$
y(k+j)=G_{j}^{*} y(k)+B^{*}\left(q^{-j}\right) F_{j}^{*} u(k+j-1) .
$$

Observe now that

$$
\begin{aligned}
& B^{*}\left(q^{-j}\right) F_{j}^{*}= \\
& =\left(b_{0}+b_{1} z^{-1}+\cdots+b_{m} z^{-m}\right)\left(1+f_{1} z^{-1}+\cdots+(11)\right. \\
& \left.+f_{j-1} z^{-j+1}\right)= \\
& =\underbrace{b_{0}}_{w_{1}}+\underbrace{\left(b_{0} f_{1}+b_{1}\right)}_{w_{2}} z^{-1}+\underbrace{\left(b_{0} f_{2}+b_{1} f_{1}+b_{2}\right)}_{w_{3}} z^{-2}+ \\
& +\cdots+\underbrace{\left(b_{0} f_{j-1}+b_{1} f_{j-2}+\cdots+b_{j-1}\right)}_{w_{j}} z^{-j+1}+ \\
& +\underbrace{\left(b_{0} f_{j}+\cdots+b_{j}\right)}_{\pi_{n+1}^{j}} z^{-j}+\cdots+\underbrace{\left(b_{0} f_{m}+\cdots+b_{m}\right)}_{\pi_{n+m}^{j}} z^{-m} .
\end{aligned}
$$




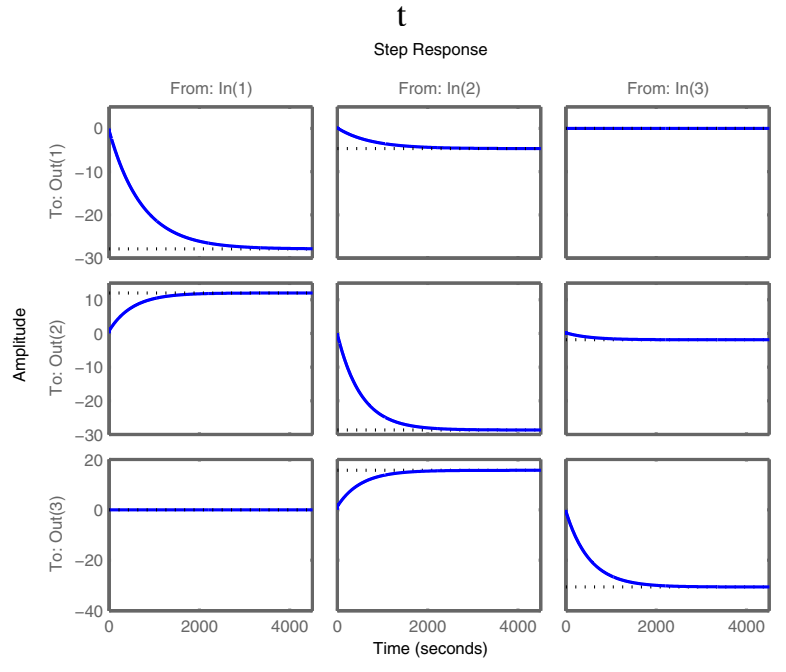

Fig. 2. Step response of the multivariable canal model considered.

and define the lower triangular Toeplitz matrix

$$
W=\left[\begin{array}{cccc}
w_{1} & 0 & \ldots & 0 \\
w_{2} & w_{1} & \ldots & 0 \\
\vdots & \vdots & \ddots & \vdots \\
w_{T} & w_{T-1} & \ldots & w_{1}
\end{array}\right]
$$

and the matrix

$$
\Pi=\left[\begin{array}{ccc}
\pi_{1}^{1} & \ldots & \pi_{n+m}^{1} \\
\vdots & \vdots & \vdots \\
\pi_{1}^{T} & \ldots & \pi_{n+m}^{T}
\end{array}\right] .
$$

The pencil of predictive models that allow to predict future outputs at time $k$ is then written

$$
\left[\begin{array}{c}
y(k+1) \\
y(k+2) \\
\cdots \\
y(k+T)
\end{array}\right]=W\left[\begin{array}{c}
u(k) \\
u(k+1) \\
\cdots \\
u(k+m)
\end{array}\right]+\Pi\left[\begin{array}{c}
y(k) \\
\cdots \\
y(k-n) \\
u(k-1) \\
\cdots \\
u(k-m+1)
\end{array}\right]
$$

\section{B. Serially connected systems}

The predictors for serially connected systems are obtained by concatenating the local predictors and considering the influence of the neighbor inputs. The step response of the multivariable canal model used in shown in figure 2. For $N_{P}=3$ the predictive model becomes

$$
Y=\left[\begin{array}{ccc}
W_{1} & W_{12} & 0 \\
W_{21} & W_{2} & W_{23} \\
0 & W_{32} & W_{3}
\end{array}\right] U+\left[\begin{array}{ccc}
\Pi_{1} & 0 & 0 \\
0 & \Pi_{2} & 0 \\
0 & 0 & \Pi_{3}
\end{array}\right] s .
$$

with

$$
Y=\left[\begin{array}{l}
\left(y_{1}\right)_{k+1}^{k+T} \\
\left(y_{2}\right)_{k+1}^{k+1} \\
\left(y_{3}\right)_{k+1}^{k+T}
\end{array}\right], \quad U=\left[\begin{array}{l}
\left(u_{1}\right)_{k}^{k+T-1} \\
\left(u_{2}\right)_{k}^{k+T-1} \\
\left(u_{3}\right)_{k}^{k+T-1}
\end{array}\right]
$$

and the state

$$
s=\left[\begin{array}{c}
\left(y_{1}\right)_{k-n+1}^{k} \\
\left(u_{1}\right)_{k-1}^{k-1} \\
\left(y_{2}\right)_{k-n+1}^{k-n} \\
\left(u_{2}\right)_{k-m}^{k-1} \\
\left(y_{3}\right)_{k-n+1}^{k} \\
\left(u_{3}\right)_{k-m}^{k-1}
\end{array}\right]
$$

In the above, the notation $x_{k_{1}}^{k_{2}} \triangleq\left[x\left(k_{1}\right) \ldots x\left(k_{1} 2\right)\right]^{T}$ is used.

\section{CENTRALIZED MPC}

The centralized MPC algorithm is obtained by minimizing $J$ with respect to all the local manipulated variables and assuming that the minimizing algorithm has access to all the input/output variables. By using the predictive model (16), the cost function becomes

$$
\begin{gathered}
J_{\text {cent }}=\|\left[\begin{array}{ccc}
W_{1} & W_{12} & 0 \\
W_{21} & W_{2} & W_{23} \\
0 & W_{32} & W_{3}
\end{array}\right] U+\left[\begin{array}{ccc}
\Pi_{1} & 0 & 0 \\
0 & \Pi_{2} & 0 \\
0 & 0 & \Pi_{3}
\end{array}\right] s- \\
-\left[\begin{array}{l}
R_{1}(k) \\
R_{2}(k) \\
R_{3}(k)
\end{array}\right]\left\|^{2}+\right\| \Omega U \|^{2},
\end{gathered}
$$

in which $\Omega$ is a block diagonal matrix of $\rho_{i} I_{T \times T}, i=1,2,3$, with $\rho_{i}$ a weight. This cost function is to be minimized in a receding horizon sense, meaning that, at each instant $k$, the cost $J_{\text {cent }}$ is minimized with respect to $U$, taking into account the constraints (e.g., by using the package cvx). Then, only the components of $U$ that correspond to the present time $k$ are actually applied to the plant, the whole procedure being repeated at the next time step.

The interest in the centralized solution consists in the fact that it provides an optimal performance bound to which decentralized solutions may be compared. Figure 3 shows the average tracking error for different values of the horizon $T$, and for the 3 local costs. For values of $T$ above 75 the closed loop system is not stable. This behavior is common to many plants controlled by MPC, being due to the fact that predictors loose their accuracy for large horizons as a consequence of modeling errors. Figure 4 shows the dependency of the cost on the weights of the manipulated variables.

\section{DISTRIBUTED MPC}

Associate to each node the cost functions

$$
\begin{gathered}
J_{1}=\left\|W_{1} U_{1}+W_{1,2} U_{2}+\Pi_{1} s_{1}-R_{1}\right\|^{2}+\rho_{1}\left\|U_{1}\right\|^{2} \\
J_{2}=\left\|W_{2} U_{2}+W_{2,1} U_{1}+W_{2,3} U_{3}+\Pi_{2} s_{2}-R_{2}\right\|^{2}+ \\
\rho_{2}\left\|U_{2}\right\|^{2}, \\
J_{3}=\left\|W_{3} U_{3}+W_{3,2} U_{2}+\Pi_{3} s_{3}-R_{3}\right\|^{2}+\rho_{3}\left\|U_{3}\right\|^{2} .
\end{gathered}
$$

Each agent involved in the minimization has an internal replica of all the control variables of the other agents. The 


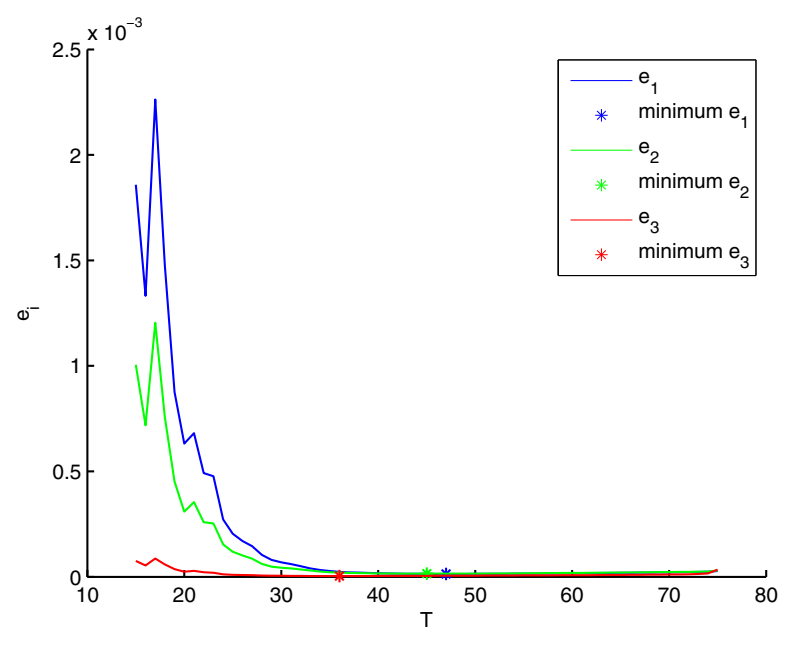

Fig. 3. Average tracking error for different values of the horizon $T$ in the range 15 to 75 , for $\rho_{1}=\rho_{2}=\rho_{3}=500$.

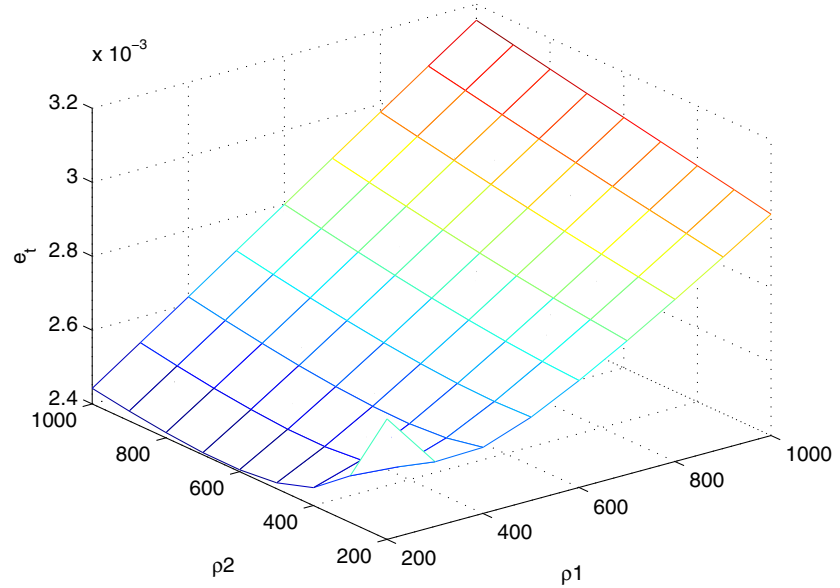

Fig. 4. Cost as a function of $\rho_{1}$ and $\rho_{2}$ for $\rho_{3}=200$ and $T=45$.

notation $U_{a}^{b}$ stands for the replica of $U_{a}$ in agent $\mathrm{b}$. With this notation, the new control variables are

$$
\begin{aligned}
& \bar{U}_{1}=\left[\begin{array}{lll}
U_{1}^{1^{T}} & U_{2}^{1^{T}} & U_{3}^{1^{T}}
\end{array}\right]^{T}, \\
& \bar{U}_{2}=\left[\begin{array}{lll}
U_{1}^{2^{T}} & U_{2}^{2^{T}} & U_{3}^{2^{T}}
\end{array}\right]^{T}, \\
& \bar{U}_{3}=\left[\begin{array}{lll}
U_{1}^{3^{T}} & U_{2}^{3^{T}} & U_{3}^{3^{T}}
\end{array}\right]^{T} .
\end{aligned}
$$

In terms of the replicated manipulated variables the local cost functions are written as

$$
\begin{aligned}
& J_{1}=\left(\bar{W}_{1} \bar{U}_{1}+Y_{10}\right)^{T}\left(\bar{W}_{1} \bar{U}_{1}+Y_{10}\right)+\bar{U}_{1}^{T} \bar{\rho}_{1} \bar{U}_{1}, \\
& J_{2}=\left(\bar{W}_{2} \bar{U}_{2}+Y_{20}\right)^{T}\left(\bar{W}_{2} \bar{U}_{2}+Y_{20}\right)+\bar{U}_{2}^{T} \bar{\rho}_{2} \bar{U}_{2}, \\
& J_{3}=\left(\bar{W}_{3} \bar{U}_{3}+Y_{30}\right)^{T}\left(\bar{W}_{3} \bar{U}_{3}+Y_{30}\right)+\bar{U}_{3}^{T} \bar{\rho}_{3} \bar{U}_{3},
\end{aligned}
$$

with

$$
\begin{gathered}
\bar{W}_{1}=\left[\begin{array}{lll}
W_{1} & W_{1,2} & 0
\end{array}\right], \\
\bar{W}_{2}=\left[\begin{array}{lll}
W_{2,1} & W_{2} & W_{2,3}
\end{array}\right], \\
\bar{W}_{3}=\left[\begin{array}{lll}
0 & W_{3,2} & W_{3}
\end{array}\right], \\
Y_{10}=\Pi_{1} s_{1}-R_{1}, \\
Y_{20}=\Pi_{2} s_{2}-R_{2}, \\
Y_{30}=\Pi_{3} s_{3}-R_{3},
\end{gathered}
$$

and

$$
\begin{gathered}
\bar{\rho}_{1}=\rho_{1}\left[\begin{array}{ccc}
I & 0 & 0 \\
0 & 0 & 0 \\
0 & 0 & 0
\end{array}\right], \quad \bar{\rho}_{2}=\rho_{2}\left[\begin{array}{lll}
0 & 0 & 0 \\
0 & I & 0 \\
0 & 0 & 0
\end{array}\right], \\
\bar{\rho}_{3}=\rho_{3}\left[\begin{array}{lll}
0 & 0 & 0 \\
0 & 0 & 0 \\
0 & 0 & I
\end{array}\right] .
\end{gathered}
$$

The minimization of $J_{1}, J_{2}$ and $J_{3}$ by the local control agents is made under the coordination constraints $\bar{U}_{1}=\bar{U}_{2}$ and $\bar{U}_{2}=\bar{U}_{3}$. The D-ADMM algorithm is implemented in this paper with the dual variables associated to the edges. In association to this algorithm, the two dual variables $\lambda$ and $\eta$ and the cost weight variable $\rho_{a d}$ are considered. As required by D-ADMM, the augmented lagrangian functions associated to the local cost functions are given by

$$
\begin{gathered}
L_{1}=J_{1}-\lambda^{T} \bar{U}_{1}+\frac{1}{2} \rho_{a d}\left\|\bar{U}_{1}-\bar{U}_{2}\right\|^{2}, \\
L_{2}=J_{2}+(\lambda-\eta)^{T} \bar{U}_{2}+\rho_{a d}\left(\left\|\bar{U}_{2}-\bar{U}_{1}\right\|^{2}+\right. \\
\left.\left\|\bar{U}_{2}-\bar{U}_{3}\right\|^{2}\right), \\
L_{3}=J_{3}+\eta^{T} \bar{U}_{3}+\frac{1}{2} \rho_{a d}\left\|\bar{U}_{3}-\bar{U}_{2}\right\|^{2},
\end{gathered}
$$

or

$$
\begin{gathered}
L_{1}=\bar{U}_{1}\left(\bar{W}_{1}^{T} \bar{W}_{1}+\bar{\rho}_{1}+\frac{\rho_{a d}}{2} I\right) \bar{U}_{1}^{T}+ \\
+\left(2 \bar{W}_{1}^{T} Y_{10}-\lambda-\rho_{a d} \bar{U}_{2}\right) \bar{U}_{1}^{T}+\epsilon_{1}, \\
L_{2}=\bar{U}_{2}\left(\bar{W}_{2}^{T} \bar{W}_{2}+\bar{\rho}_{2}+\rho_{a d} I\right) \bar{U}_{2}^{T}+ \\
+\left(2 \bar{W}_{2}^{T} Y_{20}+(\lambda-\eta)-\rho_{a d}\left(\bar{U}_{1}+\bar{U}_{3}\right)\right) \bar{U}_{2}^{T}+\epsilon_{2}, \\
L_{3}=\bar{U}_{3}\left(\bar{W}_{3}^{T} \bar{W}_{3}+\bar{\rho}_{3}+\frac{\rho_{a d}}{2} I\right) \bar{U}_{3}^{T}+ \\
+\left(2 \bar{W}_{3}^{T} Y_{30}+\eta-\rho_{a d} \bar{U}_{2}\right) \bar{U}_{3}^{T}+\epsilon_{3} .
\end{gathered}
$$

The terms in each cost function that do not depend on the minimizing variable are represented by $\epsilon_{i}$ and are not shown since they do not affect the optimal value of the argument. Therefore, for simplification purposes

$$
\begin{aligned}
& L_{1}=\bar{U}_{1} M_{1} \bar{U}_{1}^{T}+\Phi_{1} \bar{U}_{1}^{T}, \\
& L_{2}=\bar{U}_{2} M_{2} \bar{U}_{2}^{T}+\Phi_{2} \bar{U}_{2}^{T}, \\
& L_{3}=\bar{U}_{3} M_{3} \bar{U}_{3}^{T}+\Phi_{3} \bar{U}_{3}^{T},
\end{aligned}
$$


with definitions for $M_{i}$ and $\Phi_{i}$ that can be readily recovered from (42). This leads to

$$
\begin{aligned}
& \frac{\partial L_{1}}{\partial \bar{U}_{1}^{T}}=2 \bar{U}_{1} M_{1}+\Phi_{1}, \\
& \frac{\partial L_{2}}{\partial \bar{U}_{2}^{T}}=2 \bar{U}_{2} M_{2}+\Phi_{2}, \\
& \frac{\partial L_{3}}{\partial \bar{U}_{3}^{T}}=2 \bar{U}_{3} M_{3}+\Phi_{3},
\end{aligned}
$$

and the minimum for the control variables is

$$
\begin{aligned}
\bar{U}_{1}^{*} & =-\frac{1}{2} M_{1}^{-1} \Phi_{1}, \\
\bar{U}_{2}^{*} & =-\frac{1}{2} M_{2}^{-1} \Phi_{2}, \\
\bar{U}_{3}^{*} & =-\frac{1}{2} M_{3}^{-1} \Phi_{3} .
\end{aligned}
$$

Thus, upon comparison with [21], the algorithm for computing the solution using D-ADMM is as follows:

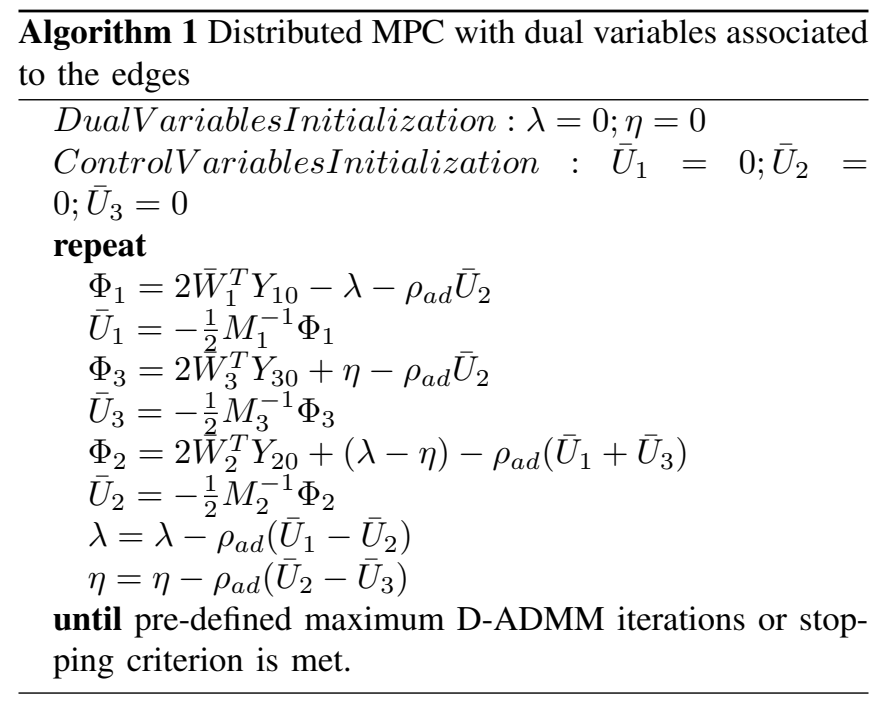

The D-ADMM algorithm is provably convergent when the network is bipartite or when the local cost functions are strongly convex (Corollary 1, [21]). Incidentally, both conditions hold in our problem: a linear graph is always bipartite, and the local cost functions (20)-(22) are strongly convex. This means that, after the execution of Algorithm 1, all agents will know the minimizer of (19). Furthermore, given that the functions in (20)-(22) are differentiable and their gradients are Lipschitz continuous, it can be shown that the primal and dual variables in Algorithm 1 converge linearly to their optimal values [22].

Applying algorithm 1, using $\rho_{a d}=100, n_{i t}=20$ (number of iterations) and $\rho_{1}=200, \rho_{2}=600, \rho_{3}=200, T=$ 45 , the simulation results shown in figures 6,7 , and 8 are obtained. As seen in figure 5, the choice made for $\rho_{a d}$ and $n_{i t}$ is the one that corresponds to the smallest computational load.

\section{CONCLUSIONS}

The use of predictive models based on input/output data dispenses the use of a state estimator.

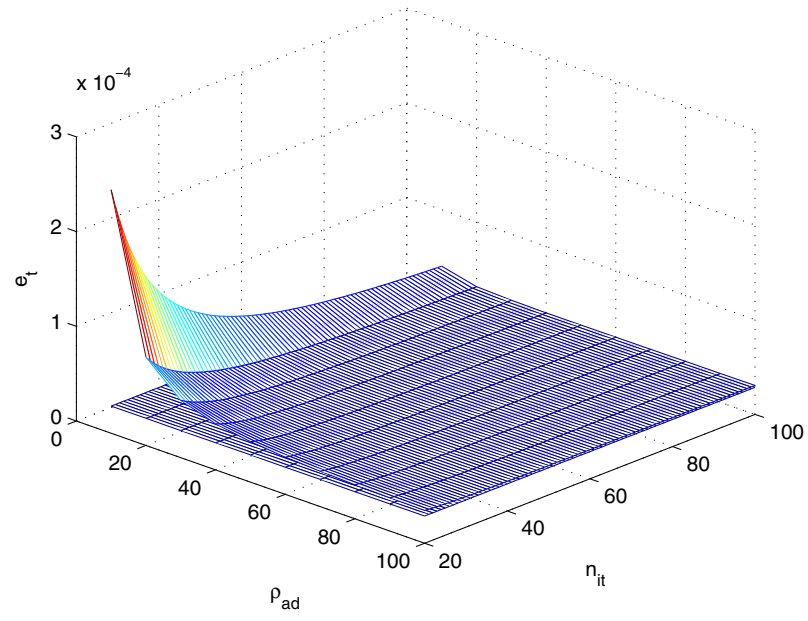

Fig. 5. Average error dependence on the D-ADMM parameters $\rho_{a d}$ and $n_{i t}$ for $\rho_{1}=200, \rho_{2}=600, \rho_{3}=200$ and $T=45$.
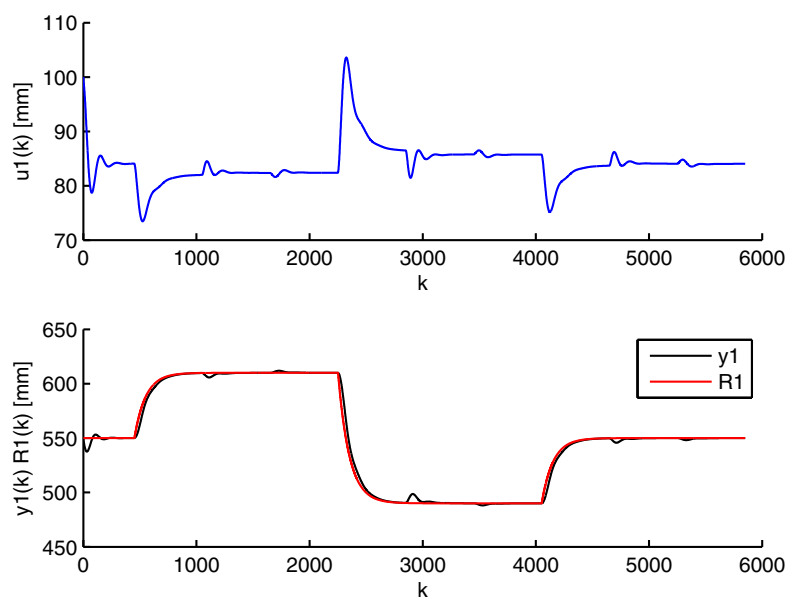

Fig. 6. Distributed control of the three pools, using D-ADMM with $\rho_{a d}=$ $100, n_{i t}=20$, and $\rho_{1}=200, \rho_{2}=600, \rho_{3}=200$ and $T=45$. Results for pool 1 .

There are different types of D-ADMM algorithms that optimize different aspects. In the situation considered in this paper, a D-ADMM algorithm with the dual variables associated to the edges has been considered. This formulation is less intuitive, but the resulting algorithm optimizes the number of messages exchanged among neighbor nodes.

The configuration of the distributed MPC algorithm comprises two parts: The configuration of the local controllers and the selection of the parameters for the D-ADMM optimizer. In what concerns the first part one has to select the horizons and the control weights. The guidelines known for centralized problems apply here, and the paper provides plots that show the influence of these parameters on the cost. A more specific remark that applies to the distributed control situation faced is that it is advantageous to use different weights in different local MPC controllers.

The parameters of the D-ADMM optimizer are the $\rho_{a d}$ 

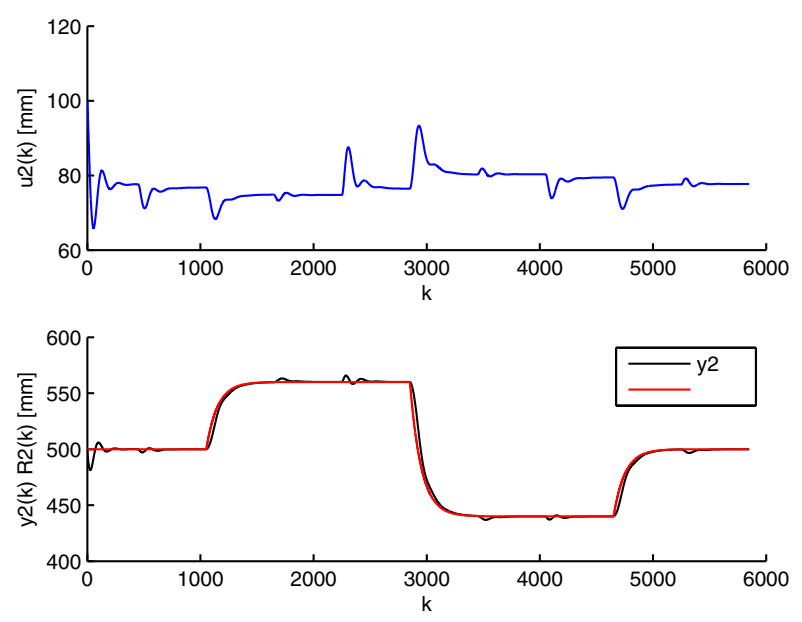

Fig. 7. Distributed control of the three pools, using D-ADMM with $\rho_{a d}=$ $100, n_{i t}=20$, and $\rho_{1}=200, \rho_{2}=600, \rho_{3}=200$ and $T=45$. Results for pool 2
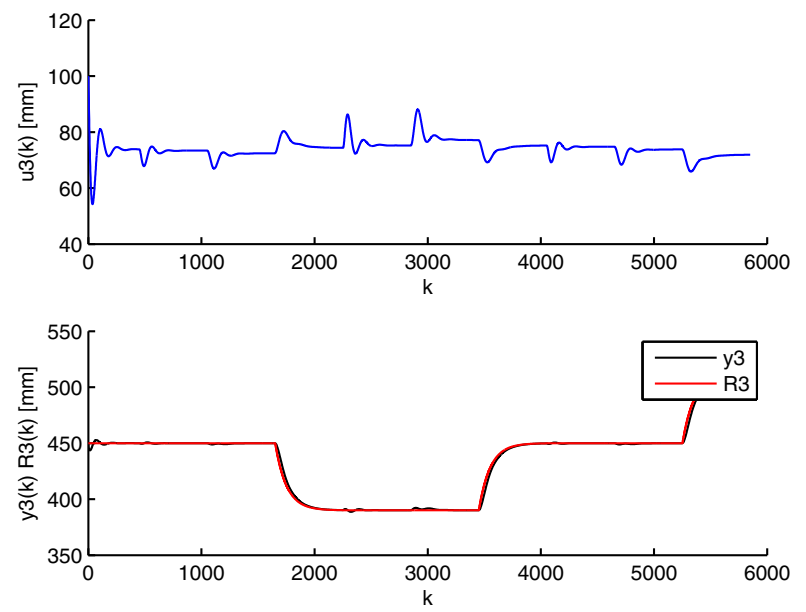

Fig. 8. Distributed control of the three pools, using D-ADMM with $\rho_{a d}=$ 100, $n_{i t}=20$, and $\rho_{1}=200, \rho_{2}=600, \rho_{3}=200$ and $T=45$. Results for pool 3 .

weight and the stopping criteria. In the paper, the main stopping criteria was the number of iterations performed, $n_{i t}$. As shown in figure 5 , the approximation of the optimum depends on an interplay between $\rho_{a d}$ and $n_{i t}$, being possible to select these parameters such as to optimize the computational load.

The final algorithm performs the optimization using closed form expressions. This is possible because the paper considers linear models together with a quadratic cost and no inequality constraints directly embedded in the optimization. Constraints are taken into consideration by a priori selecting the penalty weights of the manipulated variables in the cost functions. It is possible to modify the algorithm to incorporate constraints explicitly, but in this case one must resort to a numerical optimization algorithm, that implies a higher computational load.

\section{REFERENCES}

[1] J. M. Maestre and R. Negeenborn (eds.). Distributed Model Predictive Control Made Easy. Springer, 2014.

[2] E. Camponogara, D. Jia, B. H. Krogh, S. Talukdar (2002). Distributed model predictive control. IEEE Control Systems Magazine, 22(1):4452.

[3] T. Keviczky, F. Borrelli and G. J. Balas (2006). distributed receding horizon control for large scale dynamically decoupled systems. Automatica, 42:2105-2115.

[4] A . Richards and J. How (2007). Robust distributed model predictive control. Int. J. of Control, 80(9):1517-1531.

[5] Y. Zheng, S. Li and N. Li. Distributed model predictive control over network information exchange for large-scale systems. Control Eng. Practice, vol. 19, pp. 757-769, 2011.

[6] A. Venkat, I. Hiskens, J. Rawlings and S. Wright (2008). Distributed MPC strategies with applications to power system automatic generation control. IEEE Trans. Control Syst. Tech., 16(6):1192-1206.

[7] H. Scheu and W. Marquardt. Sensitivity-based coordination in distributed model predictive control. J. Proc. Control, vol. 21, pp. 715728, 2011.

[8] J. M. Maestre, D. Muñoz de la Peña, E. F. Camacho and T. Alamo. Distributed model predictive control based on agent negotiation. $J$. Proc. Control, vol. 21, pp. 685-697, 2011.

[9] J. Rivera, P. Wolfrum, S. Hirche, C. Goebel and H.-A. Joacobsen. Alternating Direction Method of Multipliers for Decentralized Electric Vehicle Charging Control. 52nd IEEE Conference on Decision and Control, Florence, Italy, pp. 6960-6965, 2010.

[10] Y. Zhang and S. Y. Li (2007). Networked model predictive control based on neighbourhood optimization for serially connected largescale processes. J. Process Control, 17(1): 37-50.

[11] J. M. Igreja, J. M. Lemos, F. M. Cadete, L. M. Rato and M. Rijo (2012). Control of a water delivery canal with cooperative distributed MPC. American Control Conference, Montral, Canada, pp. 3346-3351, 2012.

[12] J. M. Lemos and L. F. Pinto. Distributed Linear-Quadratic Control of Serially Chained Systems - Application to a Water Delivery Canal. IEEE Control Systems Mag., vol. 32 n. 6, pp. 26-38, 2012.

[13] A. Rantzer and K. Martensson. Gradient methods for iterative distributed control. Proc. 48th IEEE Conf. on Decision and Control, Shanghai, China, pp. 549-554, 2009

[14] Gomez, M.; J. Rodellar, and J. A. Mantecon (2002). Predictive control method for distributed operation of irrigation canals, Applied Mathematical Modelling, 26:1039-1056.

[15] R. R. Negenborn, P. J. van Overloop, T. Kevikzky, B. de Schutter (2009). Distributed model predictive control for irrigation canals Networks and Heterogeneous Media, 4(2):359-380.

[16] S. Boyd and L. Vanderberghe. Convex Optimization, Cambridge University Press, 2004.

[17] D. Gabay and B. Mercier. A dual algorithm for the solution of nonlinear variational problems via finite element approximations. Computers and Mathematics with Applications, vol. 2, pp. 1740, 1976

[18] H. El Fawal, D. Georges and G. Bonard. Optimal control of complex irrigation systems via decomposition-coordination and the use of augmented Lagrangian. Prof. IEEE Int. Conf. on Systems, Man, and Cybernetics, San Diego, CA, USA, pp. 3874-3879, 1998.

[19] S. Boyd, N. Parikh, E. Chu, B. Peleato, and J. Eckstein. Distributed Optimization and Statistical Learning via Alternating Direction Method of Multipliers, Foundations and Trends in Machine Learning, vol. 3, n. 1, pp. 1-122, 2011.

[20] J. Mota, J. Xavier, P. Aguiar and M. Püschel. Distributed ADMM for Model Predictive Control and Congestion Control. 51st IEEE Conf. on Decision and Control (CDC), pp. 5110-5115, Maui, 2012.

[21] J. Mota, J. Xavier, P. Aguiar and M. Püschel. D-ADMM: A Communication-Efficient Distributed Algorithm for Separable Optimization. IEEE Trans. on Signal Proc., vol. 16, n. 10, pp. 2718-2723.

[22] W. Deng and W. Yin. On the global and linear convergence of the generalized alternating direction method of multiplers. Tech. Rep., Rice UNiversity, Dept. Computational and Applied Mathematics, 2012. 